\title{
Multiplexed nanomedicine for brain tumors: nanosized Hercules to tame our Lernaean Hydra inside?
}

\author{
"The evolution of nanotechnologies offers \\ hope to formulate a nanosized Hercules \\ to attack brain tumor-initiating cells at \\ the core..."
}

First draft submitted: 17 August 2017; Accepted for publication: 30 August 2017; Published online: 3 October 2017

\section{Keywords: GBM $\bullet$ nanoparticle $\bullet$ nanomedicine $\bullet$ brain tumor $\bullet$ siRNA $\bullet$ convection enhanced delivery $\bullet$ BTIC/brain tumor-initiating cells $\bullet$ stem cell transcription factor}

A vivid embodiment of human fear is the gruesome monster Lernaean Hydra from Greek and Roman mythology, which is depicted as a multiheaded serpent guarding the entry to the underworld with nightmarish regenerative powers: for every chopped off head, replacements would multiply and the surging demonic ferocity would ensue (Figure 1). Similar motifs of such hopeless terror scatter across cultures, and the GrecoRoman fable teaches that divine intervention and the incredible might of the legendary Hercules were needed to kill the beast. A remarkable parallel can be drawn with our continuing, capricious combat against one of the deadliest human diseases to date: glioblastoma (GBM). With merely 14 months of average life expectancy despite maximal combination therapy of surgical resection, radiation and chemotherapy, GBM is the Hydra inside marked by persistent therapy resistance and unstoppable recurrence. Brain tumor-initiating cells (BTICs) are analogous to the regenerative venomous Hydra heads, in that existing therapeutic stress from classic therapy modalities only emboldens the aggressive progression of the disease, driven by these adaptive cell types in GBM. An obvious question is: where is our rescuer, Hercules? The evolution of nanotechnologies offers hope to formulate a nanosized Her- cules to attack BTICs at the core, the labor that inspired us to devote this editorial to elaborate the implications.

\section{Evolving nano formulations of RNAi expand therapeutic potential for heterogeneous GBM}

RNAi via safe and efficacious delivery of siRNAs is on the rise at the clinical translation front. It can directly correct aberrant gene expressions that instigate malignant tumor growth in the CNS, which can be 'undruggable' when using small molecules and biologicals (e.g., antibodies). A variety of nanosized biomaterials have been formulated to carry out this repair mission with specific therapeutic targets identified by molecular and genetic probing of the cancer genome from patient-derived tissue samples. The formulation of nano vehicles for RNAi is critical for the clinical success. Early efforts focused on dendrimer inspired nano constructs and solid nanoparticles (NPs), followed by the biomimetic development of tumor derived exosome-based experimental therapeutics for brain delivery [1-19]. The engineering potential of cell type selectivity for these vehicles is limited. Further development of novel lipopolymeric nanoparticle (LPNP) platforms incorporates engineered polymers in the lipid bilayer outer shell, allowing for flexible cel-
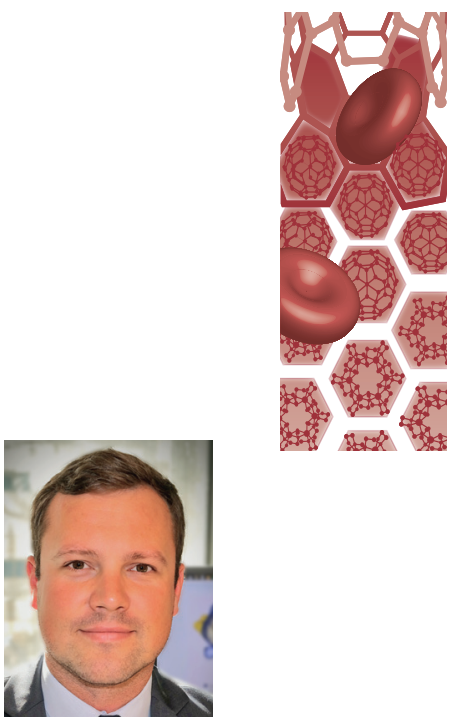

Wojciech K Panek

Department of Neurological Surgery, Brain Tumor Research Institute,

The Feinberg School of Medicine, Northwestern University, Chicago, IL 60611, USA

Omar F Khan

David $\mathrm{H}$. Koch Institute for Integrative Cancer Research, Department of Chemical Engineering, Institute for Medical Engineering \& Science, Harvard MIT Division of Health Science \&

Technology, Massachusetts Institute of Technology, Cambridge, MA 02139, USA

Dou Yu

Department of Neurological Surgery, Brain Tumor Research Institute, The Feinberg School of Medicine, Northwestern University, Chicago, IL 60611, USA

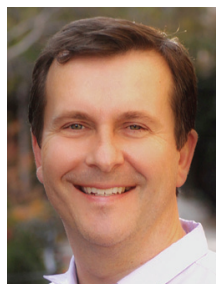

Maciej S Lesniak

Author for correspondence:

Department of Neurological Surgery, Brain Tumor Research Institute,

The Feinberg School of Medicine, Northwestern University, Chicago, IL 60611, USA

Tel.: +1 3129261094

Fax: +1 3126953294

maciej.lesniak@northwestern.edu

${ }^{\ddagger}$ Authors contributed equally

Future $\because \%$ Medicine part of 


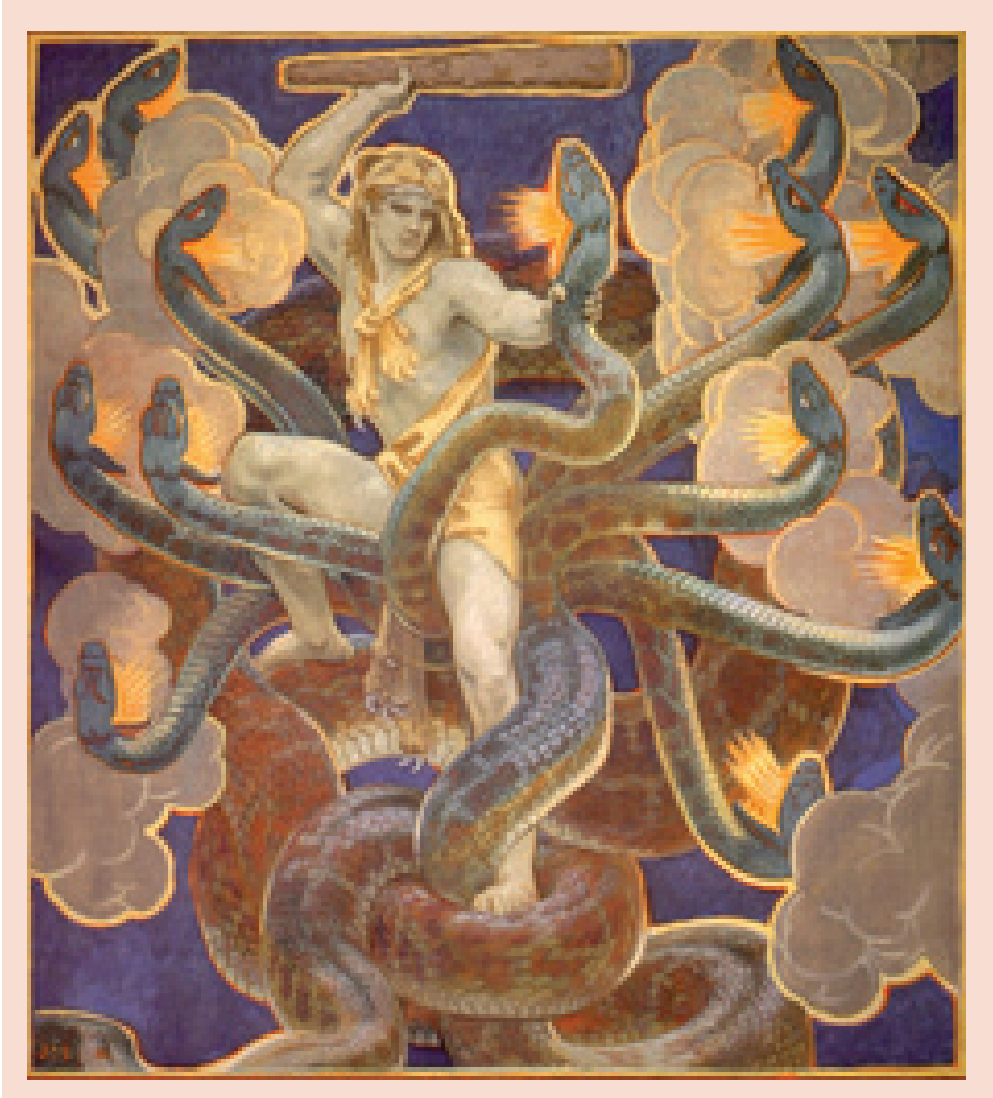

Figure 1. Hercules, by John Singer Sargent (1921), depicting the epic battle with the Lernaean Hydra. Museum of Fine Arts, Boston, MA, USA. Wikimedia Commons.

lular uptake properties [20-22]. The ability to easily modify the chemistry of the lipid NP surface was demonstrated by recent advancements $[9,23]$. In the latest article by Yu et al. [23], BTIC specific uptake of LPNPs was observed, which opens the opportunity to fine tune the tumor targeting selectivity of LPNPs, thereby enhancing therapeutic potency while minimizing off-targeting or healthy tissue toxicity.

\section{Multi-targeting capacity is vital for nanoRNAi formulations against patient specific GBM}

The evolution of nano carriers for siRNAs started with single core solid nano constructs based on classic materials such as gold [24], iron oxide, chitosan [5,17], porous silica [8], sugar [15], solid lipids [3,4,6,9] and an array of dendrimer inspired NPs [12]. Some of these applications showed early promise in effective RNAi of key GBM growth-promoting genes: Jensen et al. demonstrated that gold NP mediated siRNA knockdown of the oncoprotein Bcl2Like12 (Bcl2L12) reduced tumor growth [24]; Costa et al. demonstrated effective GBM inhibition using anti-miR-21 oligonucleotides, a different approach for tumor promoting miR-21 silencing [4]; and Danhier et al. also showed combined benefits of TMZ/chemo with chitosan based nano RNAi against Galectin and EGFR [5]. However, the most exciting potential of the latest multiplexed nano RNAi therapy against BTIC is the ability to customize the therapeutic targeting based on patient tissue features and the capacity to co-deliver synergistic multi-targeting siRNAs. Using a flexible LPNP nano carrier system, Yu et al. demonstrated sustained therapeutic benefit by incorporating four distinct siRNA constructs directed at four key master transcription factors, namely SOX2, OLIG2, SALL2 and POU3F2, in tumor growth driving BTICs [23]. The implication of this new advancement is that a variety of multiplexed RNAi therapy schemes are now possible based on an individual patient's tumor genetic profile, and efficacious personalized therapeutic outcomes are achievable even with increased evidence of intratumoral heterogeneity and post-therapy tumor metabolic adaptation. Given that diverse genetic drivers are continuously uncovered based on GBM BTIC characterization [25-27], therapeutic strategies targeting a singular genetic/epigenetic abnormality in GBM are insufficient to subdue the 'multiheaded monster.'

\section{Essential considerations for chemical engineering designs of nanoRNAi systems}

The journey towards efficacious nanomedicine for complex GBM starts with careful considerations of the nano construct design. For any nanoscale carrier delivering a therapeutic payload, form follows function. Drugs that are extremely hydrophobic are difficult to administer due to their low solubility and concomitant low bioavailability. For such payloads, a solid lipid NP or carbon nanotube construction can be advantageous. Their insoluble cores can partition the drug, and an outer hydrophilic layer, at times built using a surfactant, is used to promote cell membrane permeability. By contrast, RNAs, including siRNAs, are soluble and polyanionic. However, solubility does not mean greater bioavailability for RNA; the immune system responds to foreign RNAs as pathogenic, readily degrading them. Nucleotide chemical modifications can help prevent an immune response and degradation. Modified RNA can thus be attached to the surface of solid NPs. For example, gold particles with modified surface chemistries can enable covalent or electrostatic attachment. Charged materials do have trade-offs though, as materials with permanent charges can be more readily opsonized or toxic. Forgoing the NP paradigm and directly conjugating modified RNA to targeting moieties is another potential solution, though endosomal escape after uptake can potentially be compromised. Thus, the form of the nanoscale delivery carrier must solve these many RNA-specific challenges. 


\section{Advantages of lipopolymeric nanoRNAi formulations}

LPNPs are a promising solution. Flexible chemical synthesis means charge can be controlled. Ionizable materials, such as those containing amines, are temporarily switched to a cationic state to condense the polyanionic RNA and then returned to a neutral state. Thus, toxicity challenges caused by permanent charges are avoided. The ionizable feature is also leveraged for endosomal escape post-uptake through the proton sponge effect. Additionally, polymers can efficiently condense the RNA which, when combined with RNA chemical modification, can further prevent payload exposure and degradation. Moreover, ionizable amphiphilic polymers such as lipid polymers are both amenable to RNA payloads and NP self-assembly in aqueous environments, which obviates the need for organic solvents during production. Furthermore, NP stability can be influenced by the choice of polymer and lipid molecular weight. Even diameter can be affected, which directly impacts uptake in different tissues. Conversely, because polymer molecular weights can potentially alter the performance and characteristics of lipid polymeric NPs, their polydispersity must be carefully monitored and controlled during at-scale production.

\section{Engineering considerations for brain delivery of lipopolymeric nanoRNAi formulations}

For RNAi therapy in the brain, LPNPs offer an excellent balance of payload specificity, membrane permeability and toxicity, which can significantly boost their therapeutic index. Additionally, in terms of parenteral administration, direct injection further circumvents the challenge of crossing the blood-brain barrier after systemic delivery, which greatly focuses the therapy. However, with the large magnitude of lipid-polymer combinations possible by virtue of flexible, modular chemistry and extensive material screens may reveal new formulations that can traverse the barrier. Ionizable lipid polymeric NPs also contain and shield their RNA payloads, further avoiding localized chargerelated material-induced effects and immune reactions in the brain. Another feature of lipid polymeric NPs is the RNA payload capacity. With higher ratios of RNA:lipid polymer, NPs can carry many copies of a siRNA or a multitude of different siRNAs. This type of multiplexing is tremendously advantageous because complex therapies requiring the silencing of multiple genes become possible. Moreover, one is not limited to equimolar mixes in siRNA in multiplexes. This means payloads can be proportioned to match the prevalence and duration of the target genes and gene products.
Multiplexing may be useful in some screening applications as well. The multifunctional potential of the LPNP platform remains to be explored. Theoretically, the LPNP construct can be further customized for cell type selectivity given the differences in uptake dynamics observed for different brain cell types. Moreover, the cargo content can also include imaging reagents such as magnetic nanocore or quantum dots for in vivo and ex vivo biodistribution analysis after administration. Thus, the multimodal integration of functionality in LPNPs offers versatility and feasibility for clinical translation and real-time assessment in patients.

\section{Clinical prospects of nanomedicine for GBM \\ "Glioblastoma is the Hydra inside marked by persistent therapy resistance and unstoppable recurrence. Brain tumor-initiating cells are analogous to the regenerative venomous Hydra heads, in that existing therapeutic stress from classic therapy modalities only emboldens the aggressive progression of the disease..."}

Clinical applications of nanomedicine for malignant brain tumors are rising. Several sophisticated nano formulations of RNAi or combination therapies are at the early clinical trial phases after promising preclinical proof of concept (Clinicaltrials.gov: NCT02766699, NCT01906385, NCT03020017, NCT00944801, NCT02340156, NCT02820454, NCT00470613 and NCT02022644) [28,29]. Although all current clinical trials focus on select therapeutic targets, it is yet to be seen whether multitargeting nano therapeutic strategies will translate and deliver superior antitumor efficacy. Beyond target selection, and despite the early indications of positive safety profiles for some of the nano therapeutics, there are additional factors of divergence between the various strategies that can potentially contribute to differences in therapeutic efficacy. The most important is the delivery route. Systemic delivery is a preferred route for experimental nanomedicine, because of the ostensible advantages of repeatability, minimal invasiveness and the supposedly unique blood-brain barrier penetrance capacity of most nano carriers. However, there is still a deep chasm between these perceived benefits and the actual ability to accumulate sufficient therapeutic dose at tumor sites after systemic injections to render meaningful therapeutic efficacy. With the demonstration of dose-dependent therapeutic benefits in experimental rodent models of GBM, Yu et al. confirmed recent evidence of intratumoral convection enhanced delivery (CED) using a subcutaneously implanted osmotic pump (Alzet ${ }^{\mathrm{TM}}$, Durect Corp., CA, USA). With increasing clinical interests for direct intratumoral delivery of GBM 
therapeutics via CED [30-35], this direction forgoes some of the perceptions of systemic delivery benefits and substitutes them with the potentially efficacious local accumulation of antitumor therapeutics. Clinical evaluation of CED based nano therapeutics for GBM is underway (NCT02022644). The potential for combinatorial clinical application with standard care (surgery, temozolomide and radiation) has yet to be tested and could be even greater than the multiplexed nanoRNAi alone. However, intratumoral delivery of nano therapeutics is an evolving strategy that requires continued technological innovations to reduce complications associated with the surgical implantation of catheters or other medical devices. Significant amelioration of the pain and inconvenience associated with the surgical implantation is needed for clinical translation.

\section{Challenges ahead for advancements in nanomedicine against GBM}

The putative therapeutic potential of LPNP systems in rodent models of patient derived xenograft GBM has been demonstrated. Now, several engineering feats must be conquered to expedite clinical translation and expand the CNS applications of multiplexed LPNP therapies to other neurological disorders: The molecular mechanisms of LPNP uptake and endosomal escape need to be identified in order to enhance therapeutic efficacy of the LPNP system via engineering customization; brain delivery strategies and dis-

\section{References}

1 Agrawal A, Min DH, Singh $\mathrm{N}$ et al. Functional delivery of siRNA in mice using dendriworms. ACS Nano 3(9), 2495-2504 (2009).

2 Bronisz A, Wang Y, Nowicki MO et al. Extracellular vesicles modulate the glioblastoma microenvironment via a tumor suppression signaling network directed by miR-1. Cancer Res. 74(3), 738-750 (2014).

3 Cohen ZR, Ramishetti S, Peshes-Yaloz N et al. Localized RNAi therapeutics of chemoresistant grade IV glioma using hyaluronan-grafted lipid-based nanoparticles. ACS Nano 9(2), 1581-1591 (2015).

4 Costa PM, Cardoso AL, Custodia C, Cunha P, Pereira De Almeida L, Pedroso De Lima MC. MiRNA-21 silencing mediated by tumor-targeted nanoparticles combined with sunitinib: a new multimodal gene therapy approach for glioblastoma. J. Control. Release 207, 31-39 (2015).

5 Danhier F, Messaoudi K, Lemaire L, Benoit JP, Lagarce F. Combined anti-Galectin-1 and anti-EGFR siRNA-loaded chitosan-lipid nanocapsules decrease temozolomide resistance in glioblastoma: in vivo evaluation. Int. J. Pharm. 481(1-2), 154-161 (2015). tribution plan must be refined to ensure full therapeutic coverage of the original and recurring GBM mass; methodologies must be established for dynamic sampling and therapeutic candidate adjustments based on tumor phenotype and genotype shift in response to therapy; enable therapeutic targeting of other brain tumor cellular compositions, such as immune cells and microenvironment, etc., to comprehensively purge the tumor promoting elements; the incorporation of more sophisticated genome engineering tools such as Clustered Regularly Interspersed Short Palindromic Repeats (CRISPR) technology; and enable noninvasive telemetry through, for example, live imaging modalities. With multidisciplinary collaborative efforts converging on the creation of multiplexed and multifunctional LPNP mediated nanomedicine strategies against BTICs, the prospect of a nanosized Hercules slaying the multiheaded Hydra inside our brain is bright and encouraging.

Financial \& competing interests disclosure

This work was supported by NIH R35CA197725 (MS Lesniak), Burroughs Wellcome Collaborative Travel Grant ( $D$ Yu), the Elsa U. Pardee Foundation Grant (D Yu). The authors have no other relevant affiliations or financial involvement with any organization or entity with a financial interest in or financial conflict with the subject matter or materials discussed in the manuscript apart from those disclosed.

No writing assistance was utilized in the production of this manuscript.

6 Gujrati M, Malamas A, Shin T, Jin E, Sun Y, Lu ZR. Multifunctional cationic lipid-based nanoparticles facilitate endosomal escape and reduction-triggered cytosolic siRNA release. Mol. Pharm. 11(8), 2734-2744 (2014).

7 Han L, Zhang A, Wang H et al. Tat-BMPs-PAMAM conjugates enhance therapeutic effect of small interference RNA on U251 glioma cells in vitro and in vivo. Hum. Gene Ther. 21(4), 417-426 (2010).

8 Hasanzadeh Kafshgari M, Alnakhli M, Delalat B et al. Small interfering RNA delivery by polyethylenimine-functionalised porous silicon nanoparticles. Biomater. Sci. 3(12), 1555-1565 (2015).

9 Jin J, Bae $\mathrm{KH}$, Yang $\mathrm{H}$ et al. In vivo specific delivery of c-Met siRNA to glioblastoma using cationic solid lipid nanoparticles. Bioconjug. Chem. 22(12), 2568-2572 (2011).

10 Kaneshiro TL, Lu ZR. Targeted intracellular codelivery of chemotherapeutics and nucleic acid with a well-defined dendrimer-based nanoglobular carrier. Biomaterials 30(29), 5660-5666 (2009).

11 Katakowski M, Chopp M. Exosomes as tools to suppress primary brain tumor. Cell. Mol. Neurobiol. 36(3), 343-352 (2016). 
12 Kong L, Wu Y, Alves CS, Shi X. Efficient delivery of therapeutic siRNA into glioblastoma cells using multifunctional dendrimer-entrapped gold nanoparticles. Nanomedicine (Lond.) 11(23), 3103-3115 (2016).

13 Ofek P, Fischer W, Calderon M, Haag R, Satchi-Fainaro R. In vivo delivery of small interfering RNA to tumors and their vasculature by novel dendritic nanocarriers. FASEB J. 24(9), 3122-3134 (2010).

14 Perez-Martinez FC, Ocana AV, Perez-Carrion MD, Cena $\mathrm{V}$. Dendrimers as vectors for genetic material delivery to the nervous system. Curr. Med. Chem. 19(29), 5101-5108 (2012).

15 Sizovs A, Xue L, Tolstyka ZP et al. Poly(trehalose): sugarcoated nanocomplexes promote stabilization and effective polyplex-mediated siRNA delivery. J. Am. Chem. Soc. 135(41), 15417-15424 (2013).

16 Skog J, Wurdinger T, Van Rijn S et al. Glioblastoma microvesicles transport RNA and proteins that promote tumour growth and provide diagnostic biomarkers. Nat. Cell Biol. 10(12), 1470-1476 (2008).

17 Van Woensel M, Wauthoz N, Rosiere R et al. Development of siRNA-loaded chitosan nanoparticles targeting Galectin-1 for the treatment of glioblastoma multiforme via intranasal administration. J. Control. Release 227, 71-81 (2016).

18 Veiseh $\mathrm{O}$, Kievit FM, Liu $\mathrm{V}$ et al. In vivo safety evaluation of polyarginine coated magnetic nanovectors. Mol. Pharm. 10(11), 4099-4106 (2013).

19 Yoo B, Ifediba MA, Ghosh S, Medarova Z, Moore A. Combination treatment with theranostic nanoparticles for glioblastoma sensitization to TMZ. Mol. Imaging Biol. 16(5), 680-689 (2014).

20 Chen D, Love KT, Chen Y et al. Rapid discovery of potent siRNA-containing lipid nanoparticles enabled by controlled microfluidic formulation. J. Am. Chem. Soc. 134(16), 6948-6951 (2012).

21 Dahlman JE, Barnes $\mathrm{C}$, Khan $\mathrm{O}$ et al. In vivo endothelial siRNA delivery using polymeric nanoparticles with low molecular weight. Nat. Nanotechnol. 9(8), 648-655 (2014).

22 Dong Y, Love KT, Dorkin JR et al. Lipopeptide nanoparticles for potent and selective siRNA delivery in rodents and nonhuman primates. Proc. Natl Acad. Sci. USA 111(11), 3955-3960 (2014).

23 Yu D, Khan OF, Suva ML et al. Multiplexed RNAi therapy against brain tumor-initiating cells via lipopolymeric nanoparticle infusion delays glioblastoma progression. Proc. Natl Acad. Sci. USA 114(30), E6147-E6156 (2017).

24 Jensen SA, Day ES, Ko CH et al. Spherical nucleic acid nanoparticle conjugates as an RNAi-based therapy for glioblastoma. Sci. Transl. Med. 5(209), 209ra152 (2013).

25 Kim SH, Ezhilarasan R, Phillips E et al. Serine/threonine kinase MLK4 determines mesenchymal identity in glioma stem cells in an NF-kappaB-dependent manner. Cancer Cell 29(2), 201-213 (2016).

26 Patel AP, Tirosh I, Trombetta JJ et al. Single-cell RNAseq highlights intratumoral heterogeneity in primary glioblastoma. Science 344(6190), 1396-1401 (2014).

27 Suva ML, Rheinbay E, Gillespie SM et al. Reconstructing and reprogramming the tumor-propagating potential of glioblastoma stem-like cells. Cell 157(3), 580-594 (2014).

28 Clarke JL, Molinaro AM, Cabrera JR et al. A Phase I trial of intravenous liposomal irinotecan in patients with recurrent high-grade glioma. Cancer Chemother. Pharmacol. 79(3), 603-610 (2017).

29 Diaz MR, Vivas-Mejia PE. Nanoparticles as drug delivery systems in cancer medicine: emphasis on RNAi-containing nanoliposomes. Pharmaceuticals (Basel) 6(11), 1361-1380 (2013).

30 Chen PY, Ozawa T, Drummond DC et al. Comparing routes of delivery for nanoliposomal irinotecan shows superior antitumor activity of local administration in treating intracranial glioblastoma xenografts. Neuro Oncol. 15(2), 189-197 (2013).

31 Debinski W, Tatter SB. Convection-enhanced delivery for the treatment of brain tumors. Expert Rev. Neurother. 9(10), 1519-1527 (2009).

32 Han SJ, Bankiewicz K, Butowski NA, Larson PS, Aghi MK. Interventional MRI-guided catheter placement and real time drug delivery to the central nervous system. Expert Rev. Neurother. 16(6), 635-639 (2016).

33 Mehta AM, Sonabend AM, Bruce JN. Convection-enhanced delivery. Neurotherapeutics 14(2), 358-371 (2017).

34 Sonabend AM, Carminucci AS, Amendolara B et al. Convection-enhanced delivery of etoposide is effective against murine proneural glioblastoma. Neuro Oncol. 16(9), 1210-1219 (2014).

35 Vogelbaum MA, Aghi MK. Convection-enhanced delivery for the treatment of glioblastoma. Neuro Oncol. 17(Suppl. 2), ii3-ii8 (2015). 